\title{
Evaluación del efecto citotóxico y del daño genético de extractos estandarizados de Solanum nudum con actividad anti-Plasmodium
}

\author{
Paola García-Huertas ${ }^{1}$, Adriana Pabón ${ }^{1}$, Carlos Arias $^{2}$, Silvia Blair ${ }^{1}$ \\ 1 Grupo Malaria, Sede de Investigación Universitaria, Universidad de Antioquia, Medellín, Colombia \\ 2 Centro de la Ciencia y la Investigación Farmacéutica, Medellín, Colombia
}

Introducción. La planta Solanum nudum es ampliamente usada en la medicina tradicional del Pacífico colombiano para tratar las fiebres y la malaria, o paludismo, y se ha convertido en una fuente de nuevas moléculas promisorias.

Objetivo. Evaluar el efecto citotóxico y daño genético de extractos estandarizados de $S$. nudum en diferentes modelos celulares.

Materiales y métodos. A 66 extractos estandarizados de $S$. nudum se les evaluó la actividad antiPlasmodium in vitro en dos cepas de Plasmodium falciparum, una sensible (NF54) y otra resistente (FCB2) a la cloroquina, y la citotoxicidad en células U937 y HepG2. Se seleccionaron los extractos que presentaron actividad anti-Plasmodium y baja toxicidad, y se les estimó su efecto hemolítico en eritrocitos sanos $\mathrm{O}^{+}$, el efecto mutagénico en las cepas TA98 y TA100 de Salmonella Typhimurium y el efecto genotóxico en células U937.

Resultados. Se seleccionaron cinco extractos como promisorios (28MA1, 29MA1, 51MA1, 55MA1 y 61MA1), los cuales fueron activos en las cepas de $P$. falciparum con concentración inhibitoria 50 $\left(\mathrm{Cl}_{50}\right)$ entre 9,8 y $54,8 \mu \mathrm{g} / \mathrm{ml}$. El extracto 29MA1 fue el más selectivo para Plasmodium, con índice de selectividad de 4,4 y 14,5 para las células U937 y HepG2, respectivamente. En ningún extracto se observó efecto hemolítico a $250 \mu \mathrm{g} / \mathrm{ml}$, no causaron mutaciones en las cepas TA98 y TA100 de $S$. Typhimurium, ni generaron efectos genotóxicos en células U937.

Conclusiones. La utilización de extractos estandarizados de $S$. nudum contribuye con los trabajos encaminados al desarrollo de una nueva formulación farmacéutica para tratar la malaria a partir de productos naturales.

Palabras clave: SolanumAoxicidad, Plasmodium falciparum, malaria/terapia, extractos vegetales, seguridad.

doi: http://dx.doi.org/10.7705/biomedica.v33i1.838

\section{Evaluation of cytotoxic effect and genetic damage of standardized extracts of Solanum nudum with antiplasmodial activity}

Introduction. The plant Solanum nudum (Solanaceae) is extensively used for the treatment of malariarelated symptoms in traditional medicine practices in the Colombian Pacific. Recently, it has become a significant source of promising new molecules for developing a pharmaceutical malaria treatment.

Objective. This research aimed to evaluate the cytotoxic effect and the genetic damage of standardized extracts of $S$. nudum on different cells.

Materials and methods. Sixty six standardized S. nudum extracts were used, evaluating cytotoxicity in U937 and HepG2 cells and the antiplasmodial activity using both a chloroquine-sensitive (NF54) and a chloroquine-resistant (FCB2) strain. The hemolytic effect on healthy $\mathrm{O}^{+}$erythrocytes, the mutagenic effect on S. Typhimurium TA98 and TA100 strains and the genotoxic effect on U937 cells were evaluated. The extracts that displayed both antiplasmodial activity and low toxicity were selected.

Results. Five extracts were selected: 28MA1, 29MA1, 51MA1, 55MA1 and 61MA1. These extracts were active against $P$. falciparum with $\mathrm{IC}_{50}$ between 9.8 and $54.8 \mu \mathrm{g} / \mathrm{ml}$ and selectivity indexes were calculated between 0.9 and 4.4 , the latter for 29MA1. Also, no hemolytic effects in healthy $\mathrm{O}^{+}$

\section{Contribución de los autores:}

Paola García diseñó la investigación, realizó las pruebas experimentales, tabuló los datos, analizó los resultados obtenidos y redactó el manuscrito.

Adriana Pabón asesoró las pruebas de actividad anti-Plasmodium y análisis de los resultados, y participó en la corrección del manuscrito.

Carlos Arias Londoño cuantificó las sustancias rastreadoras en los extractos estandarizados.

Silvia Blair participó en el diseño de la investigación y en la supervisión del desarrollo de la misma; revisó y corrigió el artículo de investigación. 
erythrocytes were shown at a concentration of $250 \mu \mathrm{g} / \mathrm{ml}$, nor did they cause mutations in the TA98 and TA100 strains or generate genotoxic effects in U937cells.

Conclusion. The use of standardized extracts of $S$. nudum could contribute to the body of work aimed at developing a new pharmaceutical treatment for malaria using natural products.

Key words: SolanumAtoxicity, Plasmodium falciparum, malaria/therapy, plant extracts, safety.

doi: http://dx.doi.org/10.7705/biomedica.v33i1.838

La malaria, o paludismo, compromete la salud y el desarrollo socioeconómico de las comunidades localizadas en las regiones tropicales y subtropicales del mundo. Según la Organización Mundial de la Salud, en el año 2010, se presentaron 216 millones de casos y 655.000 muertes por esta enfermedad (1). En Colombia, se informaron 62.716 casos de malaria en el 2011, de ellos, 540 complicados, y 18 muertes, con un promedio de 105.000 casos clínicos anuales en los últimos cinco años (2). Cerca del $90 \%$ de los casos en Colombia se limitan a 70 municipios (7\% del total de municipios), con un predominio del $85 \%$ en las zonas rurales (3).

La aparición, dispersión y persistencia de la resistencia de $P$. falciparum a medicamentos antipalúdicos, principalmente a la cloroquina, han llevado a la búsqueda de nuevas alternativas farmacológicas (4-6). Una de estas alternativas son los productos naturales, ya que se estima que el $80 \%$ de la población mundial utiliza la medicina tradicional basada en plantas para el tratamiento de sus enfermedades, mediante el uso de bebidas $u$ otras formas de consumo. Además, los medicamentos antipalúdicos como los derivados de la artemisinina y la quinina, se sintetizaron a partir de compuestos aislados de plantas (7). Recientemente, se ha reportado resistencia a las artemisininas, lo cual constituye una seria amenaza para la salud, dado que en ella se basan los esquemas de tratamiento para el paludismo no complicado por $P$. falciparum (8).

Colombia es un país de gran diversidad y la medicina tradicional es una práctica común en las comunidades rurales para el tratamiento de sus enfermedades. En el municipio de Tumaco (Colombia), se utiliza la planta Solanum nudum para el tratamiento de las fiebres, entre ellas las asociadas con paludismo. Esta planta de la familia Solanaceae, es un arbusto de dos metros de altura,

\footnotetext{
Correspondencia:

Paola García-Huertas, Sede de Investigación Universitaria, Universidad de Antioquia, Calle $62 \mathrm{~N}^{\circ}$ 52-59, laboratorio 610, Medellín, Colombia

Teléfono: (574) 219 6486; fax: (574) 2196487

paolaalexandrag@gmail.com

Recibido: 09/03/12; aceptado:09/09/12
}

con flores blancas, tallo amarillo y frutos verdes, que crece como una herbácea y ha sido estudiada por el Grupo Malaria desde 1989 cuando se informó su uso como antipalúdico (9-11). A partir de extractos metanólicos y hexánicos de la planta, se han aislado la sapogenina esteroidea, diosgenona $\left(\mathrm{C}_{27} \mathrm{H}_{40} \mathrm{O}_{3}\right), y$ los esteroides $\mathrm{SN} 1\left(\mathrm{C}_{29} \mathrm{H}_{44} \mathrm{O}_{5}\right)$, SN2 $\left(\mathrm{C}_{27} \mathrm{H}_{42} \mathrm{O}_{4}\right)$, SN3 $\left(\mathrm{C}_{35} \mathrm{H}_{54} \mathrm{O}_{10}\right)$, SN4 $\left(\mathrm{C}_{33} \mathrm{H}_{52} \mathrm{O}_{9}\right), \mathrm{SN} 5\left(\mathrm{C}_{33} \mathrm{H}_{52} \mathrm{O}_{9}\right.$ isómero de SN4) y SN6 $\left(\mathrm{C}_{27} \mathrm{H}_{41} \mathrm{O}_{4}\right)(11,12)$.

Se ha encontrado que los esteroides SN1, SN2, SN3, SN4 y la diosgenona tienen prometedora actividad anti-Plasmodium in vitro, sin actividad citotóxica en células HepG2 $(5,10,13)$. Por otro lado, la evaluación in vivo de la actividad clastogénica de la diosgenona y el extracto acuoso de las hojas y tallos, utilizando la prueba de micronúcleos, mostró resultados negativos, así como la actividad mutagénica realizada a la diosgenona y a los extractos acuoso, hexánico, metanólico y diclorometano de la planta $(10,14)$. En trabajos previos se encontró que los esteroides SN2 y SN4 inhiben la invasión de esporozoítos de $P$. vivax en células HepG2 (9) y, a su vez, SN2 disminuye el número de ooquistes de $P$. vivax en Anopheles albimanus (13).

Estos trabajos han permitido entender, por un lado, que la obtención de mínimas cantidades de compuestos esteroideos requiere grandes cantidades de material vegetal, proceso poco rentable y de alto impacto ambiental, y también, que los ensayos con extractos crudos sin estandarización, no garantizan la reproducibilidad de los resultados, lo que dificulta la elaboración de un producto natural. El Grupo Malaria, viene trabajando con extractos estandarizados de $S$. nudum, preparados bajo condiciones normalizadas desde la recolección y los procesos de extracción, así como del contenido de sustancias marcadoras que son compuestos mayoritarios presentes en la planta (15). Los efectos biológicos relacionados con la actividad antipalúdica, la seguridad y los efectos colaterales de estos extractos, se desconocen; por lo tanto, en esta investigación se evaluaron en 66 extractos estandarizados, la actividad anti-Plasmodium in vitro y el efecto citotóxico en células U937, y se seleccionaron los promisorios, 
a los cuales también se les valoró su citotoxicidad en células HepG2. Con estos extractos se hizo, además, un ensayo de hemólisis para detectar la especificidad de los extractos sobre eritrocitos parasitados sin afectar los no parasitados, así como ensayos de mutagenicidad y genotoxicidad para detectar alteraciones genéticas en las células en contacto con los tratamientos.

\section{Materiales y métodos}

\section{Material vegetal y obtención de extractos estandarizados}

El material vegetal de $S$. nudum fue recolectado en la zona rural del municipio de Tumaco, Nariño. Se separó el material vegetal en cada una de sus partes aéreas (hojas, tallos y frutos) y se sometieron a un proceso de secado a temperatura ambiente $\left(25^{\circ} \mathrm{C}\right)$ durante 15 días y, al horno, con temperatura de $40^{\circ} \mathrm{C}$ durante 24 horas. El material seco se molió con tamaños de partícula de $5 \mathrm{~mm}$ y $3 \mathrm{~mm}$; posteriormente, se clasificó en lotes numerados y se seleccionaron aleatoriamente para la preparación de los extractos estandarizados. Se emplearon dos métodos de extracción: el de percolación y el de soxhlet. La extracción por percolación se hizo tres veces hasta agotamiento con los solventes: éter de petróleo, diclorometano, acetato de etilo y metanol. La extracción por soxhlet se llevó a cabo con los mismos solventes, durante cuatro y seis horas. Los extractos se sometieron a presión reducida en un rotaevaporador de vacío a $40-50{ }^{\circ} \mathrm{C}$ cada 24 horas, con el propósito de eliminar todo el solvente empleado en la extracción.

Las sustancias marcadoras empleadas para la cuantificación de los extractos fueron la diosgenona y los esteroides (SN1, SN2, SN3, SN4, SN5 y SN6), identificados previamente en la planta $S$. nudum con altos grados de pureza $(99,7 \%, 90,42 \%, 98,7 \%, 84,5 \%, 75,2 \%, 95,5 \%$ y $97,2 \%$, respectivamente) $(11,12)$. En el Centro de la Ciencia y la Investigación Farmacéutica, se determinó y cuantificó el porcentaje relativo de estas sustancias marcadoras en cada uno de los extractos, utilizando un sistema de cromatografía líquida Agilent Technologies $1200^{\text {TM }}$. Este método crea las bases para la detección de esteroides presentes en extractos de la planta $S$. nudum como un parámetro de calidad en el control fitoquímico de diferentes recolecciones y extractos estandarizados obtenidos a partir de ella. Se obtuvieron 66 extractos estandarizados de $S$. nudum codificados desde 1MA1 hasta 66MA1 (Ministerio de Agricultura), los cuales se disolvieron en dimetilsulfóxido (DMSO).

\section{Ensayo de actividad anti-Plasmodium in vitro}

Se utilizaron cultivos continuos de dos cepas de $P$. falciparum, una cepa sensible (NF54) y otra resistente (FCB2) a la cloroquina, siguiendo el método radioisotópico estandarizado en el Grupo Malaria y descrito por Desjardins, et al. (16). Se evaluaron siete concentraciones de cada extracto y cada una por duplicado. La concentración de DMSO en la primera dilución fue de $1 \%$, que se ha demostrado no es tóxica para el parásito. Como control de tratamiento, se utilizó la cloroquina y como control de crecimiento se empleó medio de cultivo. Se preparó una suspensión de glóbulos rojos parasitados con un hematocrito del $2 \%$ y una parasitemia del $1 \%$. La concentración final de la hipoxantina tritiada por pozo fue de $0,8 \mu \mathrm{Ci} / \mathrm{ml}$.

El cultivo con los tratamientos se incubó a $37^{\circ} \mathrm{C}$ durante 48 horas en atmósfera de $\mathrm{CO}_{2}$ al $5 \%, \mathrm{O}_{2}$ al $5 \%$ y de $\mathrm{N}_{2}$ al $90 \%$. El plato se congeló a $-20{ }^{\circ} \mathrm{C}$ durante 14 horas para provocar la hemólisis de los eritrocitos y la lectura del ensayo se hizo en un contador beta. Los datos se analizaron con el programa GraphPad Prism ${ }^{\mathrm{TM}}$, versión 5.01, para hallar la concentración inhibitoria $50\left(\mathrm{Cl}_{50}\right)$ y un extracto se consideró activo cuando la $\mathrm{Cl}_{50}$ antiPlasmodium fue inferior a $50 \mu \mathrm{g} / \mathrm{ml}$ (17).

\section{Ensayo de citotoxicidad in vitro}

Se utilizaron las células U937 mantenidas a $37^{\circ} \mathrm{C}$ y $\mathrm{CO}_{2}$ al $5 \%$ en medio RPMI con $10 \%$ de suplemento de suero bovino fetal (SBF) inactivado (18) y las células HepG2 mantenidas a $37^{\circ} \mathrm{C}$ y $\mathrm{CO}_{2}$ al $5 \%$ en medio DMEM, con con $10 \%$ de suplemento de suero bovino fetal inactivado (19).

La citotoxicidad de los 66 extractos estandarizados se evaluó en la línea celular U937 y, a los extractos promisorios, según criterios de selección (véase más adelante), también se les valoró su efecto tóxico en la línea celular HepG2. De forma general, las células U937 y las HepG2 se prepararon a una concentración de 200.000 células $/ \mathrm{ml}$ y se sembraron en platos de 96 pozos de fondo plano (20). Para cada ensayo se utilizaron, como control de crecimiento, células cultivadas bajo las mismas condiciones en ausencia del extracto y se evaluaron siete concentraciones seriadas de cada extracto, por triplicado, en dos ensayos independientes.

La lectura de la viabilidad celular se hizo siguiendo el método MTT, descrito por Mosmann, con algunas modificaciones hechas por el Grupo Malaria $(9,21)$. Los datos se analizaron con el programa GraphPad Prism $^{\mathrm{TM}}$, versión 5.01, para hallar la concentración 
citotóxica $50\left(\mathrm{CC}_{50}\right)$ de cada extracto. Se calculó el promedio y la desviación estándar de las dos $\mathrm{CC}_{50}$ obtenidas, y la citotoxicidad se clasificó en cuatro categorías: muy tóxicos $\left(\mathrm{CC}_{50}<10 \mu \mathrm{g} /\right.$ $\mathrm{ml})$, moderadamente tóxicos $\left(\mathrm{CC}_{50} 11-30 \mu \mathrm{g} /\right.$ $\mathrm{ml})$, levemente tóxicos $\left(\mathrm{CC}_{50} 31-50 \mu \mathrm{g} / \mathrm{ml}\right)$ y potencialmente no tóxicos $\left(\mathrm{CC}_{50}>50 \mu \mathrm{g} / \mathrm{ml}\right)$. Con el fin de estimar la selectividad del extracto por el parásito frente a células huésped (para este caso U937 y HepG2), se calculó el índice de selectividad (IS) con la siguiente fórmula: $\mathrm{IS}=\mathrm{CC}_{50}$ (citotóxica) $/$ $\mathrm{Cl}_{50}$ (anti-Plasmodium) (22).

\section{Criterios de selección de extractos promisorios}

Se consideraron extractos promisorios, aquellos extractos estandarizados que cumplieran con los siguientes criterios de actividad y citotoxicidad:

- Extractos con $\mathrm{Cl}_{50}$ anti-Plasmodium inferior a $50 \mu \mathrm{g} / \mathrm{ml}$ en las cepas NF54 o FCB2 de $P$. falciparum (17).

- Extractos con índice de selectividad en la línea celular U937, superior a 2 (22)

- Extractos que según la clasificación propuesta por el Grupo Malaria, fueran levemente tóxicos y potencialmente no tóxicos para las células U937 $(20,23)$

Las pruebas posteriores se llevaron a cabo en los extractos estandarizados que se seleccionaron como promisorios.

\section{Ensayo de hemólisis}

El efecto hemolítico de los extractos promisorios se evaluó en eritrocitos humanos normales del grupo $\mathrm{O}^{+}$, preparados al $40 \%$ en solución tampón isotónica de fosfato de sodio $(10 \mathrm{mM}, \mathrm{pH} 7,4)$. Se evaluaron siete concentraciones seriadas de los extractos por triplicado y en dos ensayos independientes. La máxima concentración valorada fue $250 \mu \mathrm{g} / \mathrm{ml}$. Como control de hemólisis basal se usaron eritrocitos tratados con solución tampón isotónica de fosfato de sodio y, como control de máxima hemólisis, se utilizó el Tween 20 al $1 \%$. La suspensión de eritrocitos con los tratamientos fue incubada a $37{ }^{\circ} \mathrm{C}$ durante una hora y la cantidad de hemoglobina liberada se determinó en un espectrofotómetro a $540 \mathrm{~nm}(24,25)$. La concentración hemolítica $50\left(\mathrm{CH}_{50}\right)$ se calculó en el programa GraphPad Prism ${ }^{\mathrm{TM}}$, versión 5.01, y el porcentaje de hemólisis se calculó con la siguiente fórmula: porcentaje de hemólisis (\%)= (tratamientosolución isotónica/tween-solución isotónica) x 100.

\section{Evaluación del efecto mutagénico}

La evaluación del efecto mutagénico de los extractos estandarizados promisorios se hizo con el test de Ames en las cepas TA98 y TA100 de Salmonella Typhimurium, las cuales detectan mutaciones por corrimientos en el marco de lectura y por sustitución de pares de bases, respectivamente. Se evaluaron cuatro concentraciones de cada extracto con base en los resultados de citotoxicidad en las líneas celulares U937 y HepG2 (cuadros 1 y 2). La CC 50 para U937 se tomó como la concentración más baja y, la $\mathrm{CC}_{50}$ para HepG2, como la más alta. La cloroquina también se incluyó en los ensayos como parámetro de comparación. Los experimentos se hicieron en presencia y ausencia de activación metabólica (fracción S9, marca Moltox ${ }^{\circledR}$ ). Se utilizó como control negativo DMSO al $50 \%$; como controles positivos directos (sin fracción S9), azida de sodio $(5 \mu \mathrm{g} / \mathrm{ml})$ y 4 nitroquinolina $(5 \mu \mathrm{g} / \mathrm{ml})$ para las cepas TA100 y TA98, respectivamente, y como control positivo indirecto (con fracción S9), 2-aminofluoreno $(0,5 \mathrm{mg} / \mathrm{ml})$ para las dos cepas.

Las bacterias fueron expuestas a los tratamientos durante 90 minutos a $37{ }^{\circ} \mathrm{C}$ y los experimentos se hicieron por duplicado en dos ensayos independientes (26). Se contaron las colonias "revertantes" (sic.) por caja y se calculó el índice de mutagenicidad (IM) con la siguiente fórmula: $\mathrm{IM}=$ promedio de colonias "revertantes" (sic.) del tratamiento / promedio de colonias "revertantes" (sic.) del control negativo.

La respuesta de mutación se consideró positiva, cuando el número de colonias "revertantes" (sic.) his- a his+ se incrementó dos veces o más con relación al control negativo ( $(\mathrm{M} \geq 2)$ y cuando se observó relación dosis-respuesta entre dosis y respuesta (27).

\section{Determinación del efecto genotóxico}

El efecto genotóxico se evaluó con el ensayo de electroforesis en gel de célula única o ensayo cometa en la línea celular U937. Se evaluaron cuatro concentraciones de los extractos, siendo la máxima $\mathrm{CC}_{50}$ la obtenida en los ensayos de citotoxicidad para la misma línea celular (cuadro 2). Se colocaron 150.000 células en contacto con los tratamientos y controles (28-29). Como control negativo se utilizó DMSO al 0,5\% y, como controles positivos, peróxido de hidrógeno $\left(\mathrm{H}_{2} \mathrm{O}_{2}\right)$ a $100 \mu \mathrm{M}$ sin activación metabólica (fracción S9) y 2-aminofluoreno, a $1 \mu \mathrm{M}$, para la activación metabólica. 
Los tratamientos se incubaron durante una a tres horas y se midió la viabilidad de las células después del tratamiento (28-30). Se tomaron $20 \mu \mathrm{l}$ de las células, las cuales se mezclaron con $80 \mu \mathrm{l}$ de agarosa de bajo punto de fusión $(0,5 \%)$ y la mezcla se colocó en placas portaobjetos, previamente impregnadas con agarosa de punto de fusión normal (1\%). Cuando la mezcla se solidificó, se colocó una tercera capa de $80 \mu \mathrm{l}$ de agarosa al 0,5\% y se procesaron dos placas por tratamiento.

Las placas se sumergieron en la solución de lisis (2,5 M NaCl, 100 mM EDTA, 10 mM Tris, 10 $\%$ DMSO $1 \%$ tritón $\mathrm{X}-100$, lauril sarcosinato de sodio al $1 \%$, pH 10 , a $4^{\circ} \mathrm{C}$ ) durante una hora. Posteriormente, las placas se colocaron por 20 minutos en el solución tampón de electroforesis (10N NaOH, 200 mM EDTA, pH 13 y a $4{ }^{\circ} \mathrm{C}$ ), al cabo de este tiempo se corrió la electroforesis a 25 $\mathrm{V}$ y $300 \mathrm{~mA}$ durante 20 minutos. Todo este proceso se llevó a cabo en oscuridad. A continuación, las placas se colocaron en la solución tampón de neutralización durante cinco minutos $(0,4 \mathrm{M}$ Tris, $\left.\mathrm{pH} 7,5,4^{\circ} \mathrm{C}\right)$ y se fijaron con metanol. La tinción se hizo con $50 \mu \mathrm{l}$ de bromuro de etidio $(0,02 \mathrm{mg} / \mathrm{ml})$ y se observaron en microscopio de fluorescencia marca Nikon a $540 \mathrm{~nm}$, en 40X (31-34). Se midió la longitud total de 40 células por placa de forma manual y los datos se presentaron en micras $(\mu \mathrm{m})$. El efecto genotóxico se evaluó por duplicado en dos ensayos independientes y se obtuvieron en total 160 mediciones por tratamiento, a los cuales se les calculó el promedio y la desviación estándar.

El tipo de daño observado en las células se clasificó en cinco categorías según la longitud de los cometas, así: 0, ausencia de daño, 0 a $26 \mu \mathrm{m}$; 1, daño bajo, 27 a $32 \mu \mathrm{m}$; 2, daño medio, 33 a 38 $\mu \mathrm{m}$; 3: daño alto, 39 a $44 \mu \mathrm{m}$; 4, daño total, más de $45 \mu \mathrm{m}(35,36)$. El resultado se consideró positivo cuando se presentó algún tipo de daño en las células y si este se incrementó en relación con la concentración del extracto.

\section{Análisis estadístico}

Los datos de las absorbancias de las pruebas de citotoxicidad y actividad anti-Plasmodium y hemolítica, se analizaron en el programa GraphPad Prism ${ }^{\mathrm{TM}}$, versión 5.01, mediante un análisis de regresión no lineal con ajuste de curva y un valor $R^{2} \geq 0,920$. Para el test de Ames, se hizo un análisis de varianza (ANOVA) de una vía, para comparar el número de colonias "revertantes" (sic.) respecto al control negativo y a la activación metabólica (fracción S9); al encontrar diferencias estadísticamente significativas, se hizo un análisis de rangos múltiples (post hoc) con la prueba de Games Howell para comparar los tratamientos con el control negativo.

Los datos obtenidos en el ensayo cometa, se evaluaron mediante un análisis de varianza (ANOVA) de dos vías, para comparar la longitud de los cometas en los diferentes tratamientos y relacionarla con la presencia o ausencia de activación metabólica (fracción S9). Al encontrar diferencias estadísticamente significativas, se analizaron los rangos múltiples (post hoc) con la prueba de Tukey, para comparar los tratamientos respecto al control negativo, así como una prueba $T$ para muestras independientes, con el fin de comparar los efectos de los tratamientos en los diferentes tiempos (una y tres horas).Todas las pruebas se hicieron con un valor $p<0,05$ y se utilizó el paquete estadístico SPSS Statistics ${ }^{\mathrm{TM}}$, versión 17.0 .

\section{Resultados}

\section{Actividad citotóxica y anti-Plasmodium de extractos estandarizados promisorios de Solanum nudum}

De acuerdo con la clasificación de citotoxicidad presentada en la metodología $(20,23)$, se encontró que 28 extractos estandarizados eran muy tóxicos $(42,2 \%) ; 16$, moderadamente tóxicos $(24,2 \%) ; 12$, levemente tóxicos $(18,2 \%)$, y 10 , potencialmente no tóxicos (15,2\%). Según los criterios de selección de extractos promisorios, se escogieron cinco: 28MA1, 29MA1, 51MA1, 55MA1 y 61MA1. Los extractos 28MA1, 51MA1, 55MA1 y 61MA1 fueron catalogados como levemente tóxicos y, el extracto 29MA1, como potencialmente no tóxico. Estos extractos se obtuvieron de las hojas de $S$. nudum, extraídos por percolación y Soxhlet, y se procesaron, principalmente, con metanol (cuadro 1).

En el cuadro 1 se presentan los resultados cuantitativos de las sustancias marcadoras (esteroides) de cada extracto estandarizado y en este perfil se observa que los extractos con mayor actividad anti-Plasmodium ( $\mathrm{Cl}_{50}$ más bajas) y con mayor toxicidad $\left(\mathrm{CC}_{50}\right.$ más bajas), presentaron las mayores concentraciones de la sapogenina esteroidea diosgenona y presencia del esteroide $\mathrm{SN}-1$ a una concentración cercana al $2 \%$. En todos los extractos, excepto el 55MA1, se identificaron y cuantificaron los siete compuestos esteroideos.

Los resultados de actividad anti-Plasmodium (cuadro 1) y citotoxicidad en células U937 (cuadro 
2), mostraron que los extractos 55MA1 y 61MA1 fueron selectivos para Plasmodium (IS $>2$ ) en las dos cepas evaluadas (FCB-2 y NF-54), mientras que los extractos 28MA1, 29MA1 y 51MA1 fueron selectivos solo para la cepa resistente a la cloroquina (FCB-2) (cuadro 2).

Las células HepG2 fueron menos sensibles al efecto citotóxico de los extractos, ya que los cinco extractos estandarizados promisorios fueron potencialmente no tóxicos y los índices de selectividad fueron mayores de 2 para las dos cepas de $P$. falciparum evaluadas (cuadro 2).

\section{Efecto hemolítico de extractos estandarizados promisorios de Solanum nudum}

Los cinco extractos estandarizados seleccionados (28MA1, 29MA1, 51MA1, 55MA1 y 61MA1) no presentaron actividad hemolítica sobre los eritrocitos sanos. Los resultados fueron de $0 \%$ de hemólisis y la $\mathrm{CH}_{50}$ fue mayor de $250 \mu \mathrm{g} / \mathrm{ml}$, que fue la máxima concentración evaluada.

\section{Efecto mutagénico de extractos estandarizados de Solanum nudum en cepas de Salmonella Typhimurium}

Los resultados obtenidos en el test de Ames muestran que los extractos estandarizados promisorios no presentaron efecto mutagénico, aun en presencia y ausencia de activación metabólica, utilizando las cepas TA98 y TA100 de $S$. Typhimurium ya que no se encontró diferencia estadísticamente significativa entre el número de colonias "revertantes" (sic.) obtenido en el control negativo (DMSO) y los tratamientos (Games Howell, $p>0,05)$, ni diferencias estadísticamente significativas entre el número de colonias "revertantes" (sic.) obtenidos para los tratamientos, en las dos cepas bacterianas evaluadas (ANOVA de una vía $p>0,05$ ).

El índice de mutagenicidad para la cepa TA98 fue de 1 en todos los tratamientos y aquellos para la cepa TA100 fueron similares (figuras 1 A y B); por lo tanto, se consideró que los extractos no causaron mutaciones por corrimientos en el marco de lectura o sustitución de pares de bases, detectados por las cepas TA98 y TA100, respectivamente.

\section{Genotoxicidad de extractos de Solanum nudum en la línea celular U-937}

La viabilidad de las células después de la incubación con los tratamientos, fue superior al $95 \%$ en todos los casos, lo que indica que la concentración del tratamiento utilizado no afectó los resultados obtenidos. Los extractos

Cuadro 1. Condiciones de preparación, perfil de las sustancias marcadoras y actividad anti-Plasmodium de los extractos estandarizados de $S$. nudum seleccionados como promisorios

\begin{tabular}{|c|c|c|c|c|c|c|c|c|c|c|c|c|}
\hline \multirow[t]{2}{*}{ Código } & \multicolumn{3}{|c|}{ Extracción } & \multicolumn{7}{|c|}{ Esteroides (\%) } & \multicolumn{2}{|c|}{$\begin{array}{c}\text { Actividad anti- } \\
\text { Plasmodium } \mathrm{Cl}_{50} \pm \mathrm{DE}\end{array}$} \\
\hline & Solvente & Método & Tiempo & SN1 & SN2 & SN3 & SN4 & SN5 & SN6 & Diosg. & FCB-2 & NF-54 \\
\hline 28 MA1 & $\mathrm{MeOH}$ & Percolación & 10 días & 0,19 & 1,16 & 2,2 & 8,1 & 9,3 & 0,02 & 0,06 & $21,8 \pm 4$ & $54,8 \pm 8,1$ \\
\hline 29 MA1 & $\mathrm{MeOH}$ & Percolación & 10 días & 0,58 & 1,34 & 1,27 & 4,23 & 0,77 & 0,19 & 0,55 & $12,8 \pm 0,4$ & $35,7 \pm 4,4$ \\
\hline $51 \mathrm{MA} 1$ & EtoAc & Soxhlet & 10 días & 2,55 & 9 & 0,88 & 1,09 & 0,2 & 2,1 & 7,3 & $13,6 \pm 2$ & $20,4 \pm 2,7$ \\
\hline 55 MA1 & DCM : EtoAc (1:1) & Soxhlet & 4 horas & 2,2 & 0 & 0,7 & 0 & 0 & 2,4 & 8,8 & $14,6 \pm 1,6$ & $12,7 \pm 0,3$ \\
\hline $61 \mathrm{MA1}$ & MeOH:agua (80:20) & Soxhlet & 4 horas & 2,26 & 2,15 & 2,51 & 3,81 & 1,11 & 1,13 & 2,45 & $9,8 \pm 1,1$ & $16,9 \pm 3,3$ \\
\hline
\end{tabular}

$\mathrm{Cl}_{50}$ : concentración inhibitoria $50(\mu \mathrm{g} / \mathrm{ml})$; FCB-2: cepa de $P$. falciparum resistente a cloroquina, NF-54: cepa de $P$. falciparum sensible a cloroquina; Diosg.: diosgenona

Cuadro 2. Citotoxicidad e índice de selectividad de los extractos promisorios estandarizados de S. nudum

\begin{tabular}{|c|c|c|c|c|c|c|}
\hline \multirow{3}{*}{ Extractos } & \multicolumn{3}{|c|}{ Células U937 } & \multicolumn{3}{|c|}{ Células HepG2 } \\
\hline & \multirow{2}{*}{$\begin{array}{c}\text { Citotoxicidad } \\
\mathrm{CC}_{50} \pm \mathrm{DE}\end{array}$} & \multicolumn{2}{|c|}{ IS } & \multirow{2}{*}{$\begin{array}{c}\text { Citotoxicidad } \\
\mathrm{CC}_{50} \pm \mathrm{DE}\end{array}$} & \multicolumn{2}{|c|}{ IS } \\
\hline & & FCB-2 & NF-54 & & FCB-2 & NF-54 \\
\hline $28 \mathrm{MA} 1$ & $49,3 \pm 7,1$ & 2,3 & 0,9 & $185,9 \pm 2,3$ & 8,5 & 3,4 \\
\hline 29MA1 & $56,7 \pm 5,2$ & 4,4 & 1,6 & $185,5 \pm 35,2$ & 14,5 & 5,2 \\
\hline $51 \mathrm{MA} 1$ & $37,6 \pm 2,9$ & 2,8 & 1,8 & $70,7 \pm 4,8$ & 5,2 & 3,5 \\
\hline $55 \mathrm{MA} 1$ & $36,7 \pm 6,7$ & 2,5 & 2,9 & $85,1 \pm 5,7$ & 5,8 & 6,7 \\
\hline $61 \mathrm{MA} 1$ & $35,9 \pm 2,4$ & 3,7 & 2,1 & $61,9 \pm 2,5$ & 6,3 & 3,7 \\
\hline
\end{tabular}

$\mathrm{CC}_{50}$ : concentración citotóxica $50(\mu \mathrm{g} / \mathrm{ml})$; IS: índice de selectividad, $\mathrm{CC}_{50}$ (citotóxica)/ $\mathrm{Cl}_{50}$ (anti-Plasmodium); DE: desviación estándar; FCB-2: cepa de $P$. falciparum resistente a cloroquina; NF-54: cepa de $P$. falciparum sensible a cloroquina 

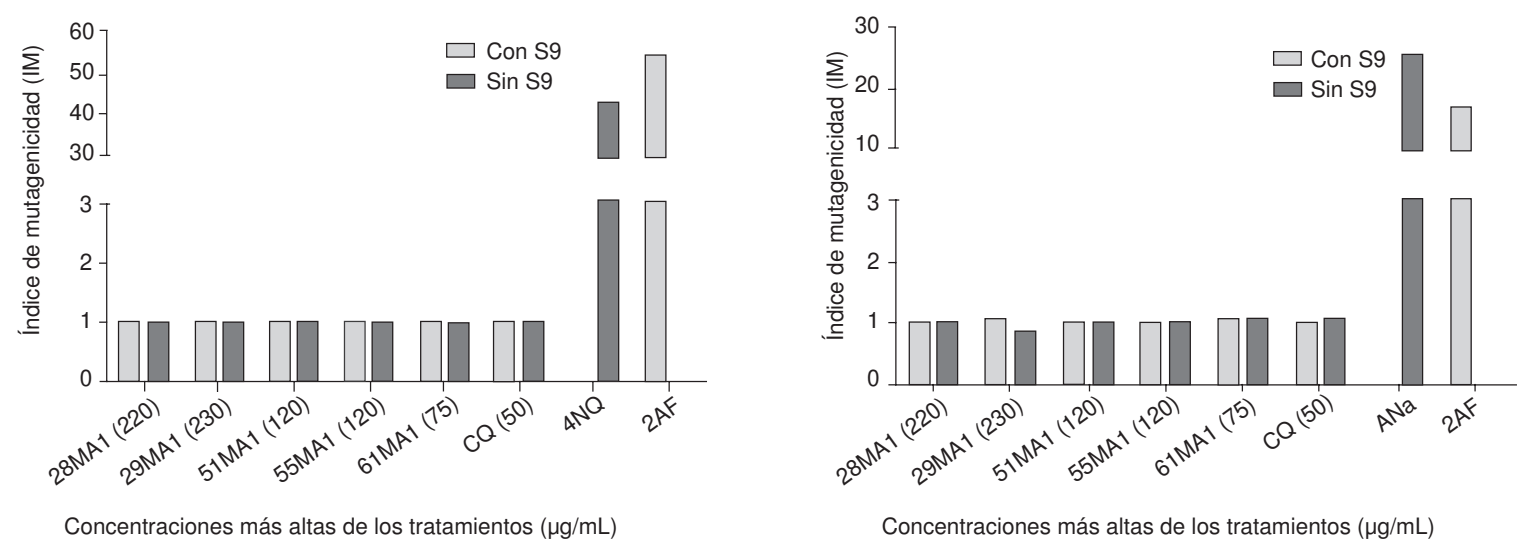

Figura 1. Índice de mutagenicidad de cada uno de los extractos a la concentración más alta ensayada con las cepas TA98 (A) y TA100 (B) de $S$. Typhimurium, con y sin activación metabólica.
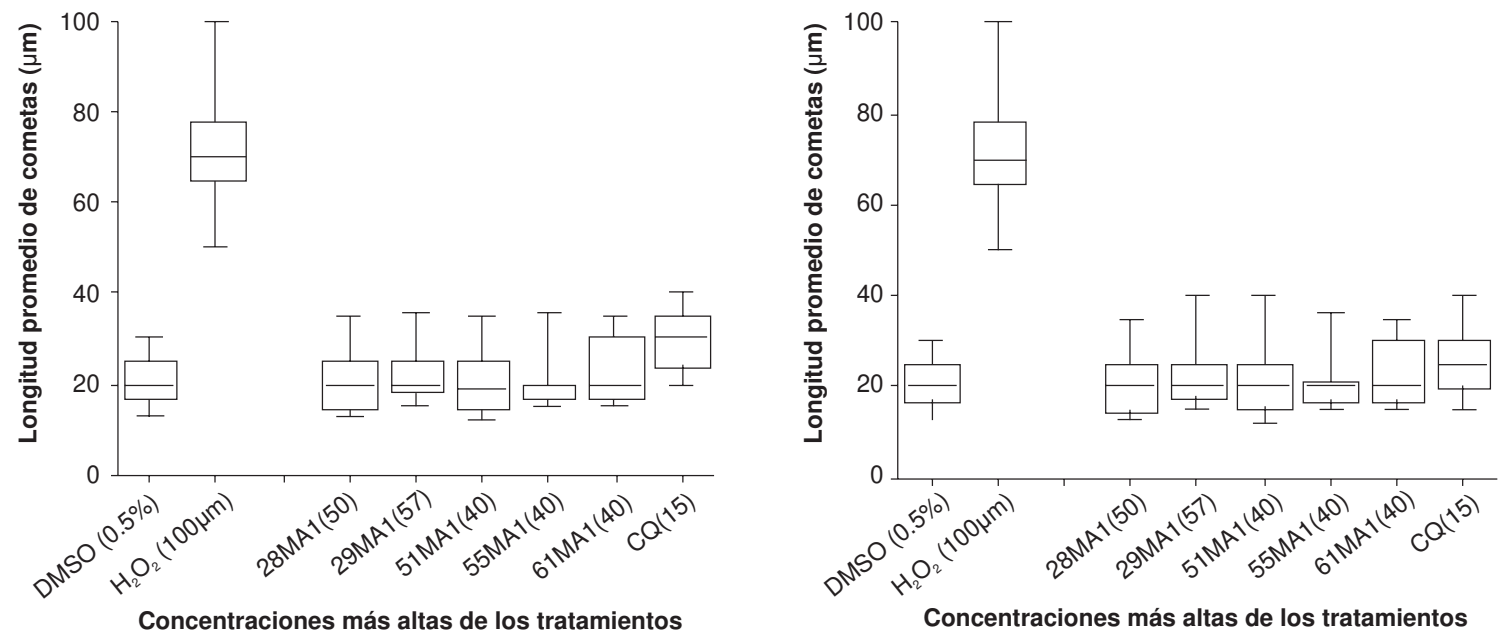

Figura 2. Longitud promedio de cometas obtenidos en células U937 después del tratamiento con las concentraciones más altas de cada extracto estandarizado. A. Una hora de tratamiento. B. Tres horas de tratamiento.

estandarizados de $S$. nudum no causaron efectos genotóxicos en las células U937 durante una o tres horas de tratamiento. No se encontraron diferencias significativas entre la longitud de los cometas de los tratamientos y el control negativo (Tukey $p<0,05)$ y la categoría de daño fue 0 (ausencia de daño) para todos los tratamientos (figuras $2 \mathrm{~A}$ y $2 \mathrm{~B}$ ). Aun al adicionar activación metabólica a los tratamientos en cada uno de los tiempos de incubación, no se encontró un efecto genotóxico (no se presentan los datos).

\section{Discusión}

Existen pocos estudios en los que se evalúen extractos estandarizados de plantas como opción terapéutica. La mayoría evalúa extractos crudos no estandarizados que no garantizan la reproducibilidad de sus efectos terapéuticos, o compuestos puros que requieren altos costos para su obtención y grandes cantidades de material vegetal. Este es el primer estudio con extractos estandarizados de $S$. nudum y los resultados obtenidos muestran que cinco de estos conservan la seguridad y actividad anti-Plasmodium reportada previamente con extractos crudos y compuestos de la misma planta.

Los extractos seleccionados de S. nudum (28MA1, 29MA1, 51MA1, 55MA1, 61MA1) tuvieron como característica común su origen a partir de hojas de plantas silvestres del municipio de Tumaco. De acuerdo con estos resultados, se puede inferir que las moléculas relacionadas con la actividad y la falta de toxicidad para las células U937 y HepG2, están concentradas en las hojas. Los efectos terapéuticos de las plantas se pueden atribuir a la gran diversidad 
de metabolitos secundarios que poseen, los cuales pueden ser utilizados por la planta para mediar su interacción ecológica con el ambiente, al actuar como defensa contra predadores y patógenos, como agentes alelopáticos 0 , incluso, para atraer a los polinizadores y dispersores de las semillas $(37,38)$.

Se encontraron diferencias en la citotoxicidad de los extractos estandarizados seleccionados como promisorios en las células U937 y HepG2, siendo las células U937 más sensibles que las HepG2. Es importante tener en cuenta que las células HepG2, son aptas para el metabolismo oxidativo, contrario a las células U937 que provienen de células sanguíneas (39). Además, las células HepG2 expresan los citocromos CYP1A1, 1A2, 1B1, 2A6, $2 \mathrm{~A} 7,2 \mathrm{~A} 13,2 \mathrm{C} 9,2 \mathrm{D} 6,2 \mathrm{E} 1,3 \mathrm{~A} 4,3 \mathrm{~A} 5$ y $3 \mathrm{~A} 7$; por el contrario, las células U937 expresan solo algunos, CYP1A1, 1A2, 1B1, 2A6, 2A7, 2D6 y 2E1(40). Por lo anterior, es importante hacer la tamización de citotoxicidad en diferentes modelos celulares provenientes de órganos que se puedan afectar por el uso de la nueva formulación en estudio.

Con respecto a la cuantificación de sustancias marcadoras para la preparación de extractos estandarizados de productos naturales, es importante anotar que la identificación de los mejores perfiles y su reproducibilidad permite seleccionar la materia prima para las evaluaciones biológicas y para el posible escalamiento de los productos activos. En nuestro caso, para observar la relación entre el perfil de esteroides y la actividad biológica, es conveniente analizar todos los extractos estandarizados, ya que cinco extractos es poco para este análisis global.

Por otro lado, la carencia de efectos hemolíticos de los extractos, bajo las condiciones evaluadas en el ensayo de hemólisis, corrobora la selectividad de estos extractos para Plasmodium. Estos datos apoyan resultados previos obtenidos por López, et al., en el 2009, quienes encontraron que los esteroides aislados de $S$. nudum y la diosgenona, no causaron hemólisis en eritrocitos sanos, pero sí en eritrocitos parasitados (24).

Se encontraron resultados igualmente prometedores en los ensayos de mutagenicidad con el test de Ames, ya que los extractos no causaron efectos mutagénicos en las cepas TA98 y TA100 de $S$. Typhimurium, lo que indica que los extractos obtenidos de la planta S. nudum carecen de efectos mutagénicos directos e indirectos (activados por enzimas) sobre las cepas empleadas y bajo las condiciones establecidas. Estos resultados se corroboran con hallazgos previos en los cuales la diosgenona y los extractos acuoso, hexánico, metanólico y de diclorometano de esta planta, no mostraron efectos mutagénicos en cepas TA97a, TA98, TA100 y TA102 (26).

Los extractos promisorios de S. nudum tampoco fueron genotóxicos para las células U937, por una y tres horas de exposición a los tratamientos. Aun la adición de activación metabólica a los tratamientos, no alteró los resultados, lo que demuestra que este comportamiento no se debe a carencias enzimáticas en la maquinaria metabólica de estas células que no hayan sido suficientes para la transformación de los extractos y la detección de agentes genotóxicos. Resultados similares fueron encontrados por Álvarez, et al., en el 2004, quienes utilizaron el ensayo de micronúcleos para evaluar el efecto clastogénico de la diosgenona y el extracto acuoso de $S$. nudum, y encontraron que tampoco causaron resultados positivos (14).

En conclusión, con este trabajo se identificaron cinco extractos estandarizados de $S$. nudum, los cuales fueron valorados como promisorios para el tratamiento del paludismo, fueron selectivos para Plasmodium, no causaron toxicidad a las células U937, HepG2 y eritrocitos sanos, y no tuvieron efectos genotóxicos en células U937 ni efectos mutagénicos en las cepas TA98 y TA100 de $S$. Typhimurium.

Los resultados obtenidos en este trabajo contribuyen a la búsqueda de nuevas opciones terapéuticas seguras para tratar la malaria con base en productos naturales y en el aprovechamiento de los recursos botánicos, en los que nuestro país es especialmente rico. Además, apoyan resultados previos donde muestran que la planta $S$. nudum puede ser una alternativa terapéutica efectiva, segura, natural y al alcance de las personas que padecen malaria. Sin embargo, se debe continuar con estudios in vivo para confirmar los promisorios resultados presentados en modelos in vitro, y hacer un análisis global de todos los extractos estandarizados para identificar y reproducir las condiciones que permitan la obtención de un extracto promisorio.

\section{Agradecimientos}

Agradecemos especialmente al Grupo Malaria de la Universidad de Antioquia, al Programa de Sostenibilidad 2011-2012 de la Universidad de Antioquia y al Ministerio de Agricultura, por la 
financiación del proyecto "Valoración de la actividad antimalárica (sic.) de extractos estandarizados obtenidos de la planta Solanum nudum nativa y cultivada in vitro" que permitió la elaboración de este trabajo. También, agradecemos a Claudia Barbosa, Isabel Cristina Ortiz, Omar Triana y Ana María Mesa, así como al Centro de la Ciencia y la Investigación Farmacéutica (CECIF).

\section{Conflicto de interés}

Todos los autores de este documento declaran que no existe conflicto de interés.

\section{Financiación}

Este trabajo fue financiado por el Ministerio de Agricultura de Colombia, con el proyecto "Valoración de la actividad antipalúdica de extractos estandarizados obtenidos de la planta Solanum nudum nativa y cultivada in vitro" (0092007UV7552-2907), por el Grupo Malaria y el Programa de Sostenibilidad 2011-2012 de la Universidad de Antioquia.

\section{Referencias}

1. World Health Organization. World Malaria Report2011. Fecha de consulta: 4 de julio de 2012. Disponible en:http://www.who.int/malaria/world_malaria_report_ 2011/9789241564403_eng.pdf.

2. Instituto Nacional de Salud. Boletín epidemiológico semanal, semana 52-2011. Fecha de consulta: 4 de julio de 2012. Disponible en: http://www.ins.gov.co/boletinepidemiologico/Boletn\%20Epidemiolgico/2011\%20 Boletin\%20epidemiologico_Semana\%2052.pdf.

3. Rodríguez JC, Uribe GA, Araújo RM, Narváez PC, Valencia SH. Epidemiology and control of malaria in Colombia. Mem Inst Oswaldo Cruz. 2011;106:114-22. http:// dx.doi.org/10.1590/S0074-02762011000900015

4. Arango E, Carmona J, Blair S. Susceptibilidad in vitro de muestras clínicas colombianas de Plasmodium falciparum a tres esteroides de la planta Solanum nudum dunal (Solanaceae). Vitae. 2008;15:150-6.

5. Arango E, Carmona J, Blair S. Susceptibilidad in vitro de aislamientos colombianos de Plasmodium falciparum a diferentes antipalúdicos. Biomédica. 2008;28:213-23.

6. Blair S, Lacharme LL, Fonseca JC, Tobón A. Resistance of Plasmodium falciparum to 3 antimalarials in Turbo (Antioquia, Colombia), 1998. Rev Panam Salud Pública. 2001;9:23-9. http://dx.doi.org/10.1590/S1020-49892001000100006

7. Lee MR. Plants against malaria, part 2: Artemisia annua (Qinghaosu or the sweet wormwood). J R Coll Physicians Edinb. 2002;32:300-5.

8. Dondorp AM, Yeung S, White L, Nguon C, Day NP, Socheat D, et al. Artemisinin resistance: Current status and scenarios for containment. Nat Rev Microbiol. 2010;8:27280. http://dx.doi.org/10.1038/nrmicro2331

9. Londoño B, Arango E, Zapata C, Herrera S, Saez J, Blair $\mathrm{S}$, et al. Effect of Solanum nudum Dunal (Solanaceae) steroids on hepatic trophozoites of Plasmodium vivax. Phytother Res. 2006;20:267-73. http://dx.doi.org/10.1002/ ptr.1849

10. Pabon A, Carmona J, Maestre A, Camargo M, Blair S. Inhibition of $P$. falciparum by steroids isolated from Solanum nudum. Phytother Res. 2002;16:59-62. http://dx.doi. org/10.1002/ptr.1035

11. Saez J, Cardona W, Espinal D, Blair S, Mesa J, Bocar M, et al. Five new steroids from Solanum nudum. Tetrahedron. 1998;54:10771-8. http://dx.doi.org/10.1016/S0040-4020(98) 00632-2,

12. Blair S, Mesa J, Carmona J, Saez J. Apertura del anillo $\mathrm{f}$ de la diosgenona y actividad antimalárica in vitro de los productos de reacción. Rev Colom Quim. 2001;30:97-107.

13. Arango E, Londono B, Segura C, Solarte Y, Herrera S, Saez J, et al. Prevention of sporogony of Plasmodium vivax in Anopheles albimanus by steroids of Solanum nudum Dunal (Solanaceae). Phytother Res. 2006;20:444-7. http:// dx.doi.org/10.1002/ptr.1874

14. Álvarez G, Pabón A, Carmona J, Blair S. Evaluation of clastogenic potential of the antimalarial plant Solanum nudum. Phytother Res. 2004;18:845-8.

15. Gurib-Fakim A. Medicinal plants: Traditions of yesterday and drugs of tomorrow. Mol Aspects Med. 2006;27:1-93. http://dx.doi.org/10.1002/ptr.1534

16. Desjardins RE, Canfield CJ, Haynes JD, Chulay JD. Quantitative assessment of antimalarial activity in vitro by a semiautomated microdilution technique. Antimicrob Agents Chemother. 1979;16:710-8. http://dx.doi.org/10.1128/ AAC.16.6.710

17. Jonville MC, Kodja H, Humeau L, Fournel J, De Mol P, Cao $\mathbf{M}$, et al. Screening of medicinal plants from Reunion Island for antimalarial and cytotoxic activity. J Ethnopharmacol. 2008;120:382-6. http://dx.doi.org/10.1016/j.jep.2008.09.005

18. Moore GE, Gerner RE, Franklin HA. Culture of normal human leukocytes. JAMA. 1967;199:519-24.

19. Tavakkol-Afshari J, Brook A, Mousavi SH. Study of cytotoxic and apoptogenic properties of saffron extract in human cancer cell lines. Food Chem Toxicol. 2008;46:3443-7. http://dx.doi.org/10.1016/j.fct.2008.08.018

20. Osorio E, Arango GJ, Jiménez N, Alzate F, Ruiz G, Gutiérrez D, et al. Antiprotozoal and cytotoxic activities in vitro of Colombian Annonaceae. J Ethnopharmacol. 2007;111:630-5. http://dx.doi.org/10.1016/j.jep.2007.01.015

21. Mosmann T. Rapid colorimetric assay for cellular growth and survival: Application to proliferation and cytotoxicity assays. J Immunol Methods. 1983;65:55-63. http://dx.doi. org/10.1016/0022-1759(83)90303-4,

22. Koch A, Tamez P, Pezzuto J, Soejarto D. Evaluation of plants used for antimalarial treatment by the Maasai of Kenya. J Ethnopharmacol. 2005;101:95-9. http://dx.doi. org/10.1016/j.jep.2005.03.011

23. Kaou AM, Mahiou-Leddet V, Hutter S, Ainouddine S, Hassani S, Yahaya I, et al. Antimalarial activity of crude extractsfromnineAfrican medicinal plants. JEthnopharmacol. 2008;116:74-83. http://dx.doi.org/10.1016/j.jep.2007.11.001

24. López ML, Blair S, Saez J, Segura C. Effect of Solanum nudum steroids on uninfected and Plasmodium 
falciparum-infected erythrocytes. Mem Inst Oswaldo Cruz. 2009;104:683-8. http://dx.doi.org/10.1590/S007402762009000500003

25. Okoli CO, Akah PA. Mechanisms of the anti-inflammatory activity of the leaf extracts of Culcasia scandens P. Beauv (Araceae). Pharmacol Biochem Behav. 2004;79:473-81. http://dx.doi.org/10.1016/j.pbb.2004.08.012

26. Pabón A, Blair S, Carmona J, Zuleta M, Saez J. Evaluation of the mutagenicity of antimalarial products isolated from Solanum nudum (Solanaceae). Pharmazie. 2003;58:263-7.

27. Maron DM, Ames BN. Revised methods for the Salmonella mutagenicity test. Mutat Res. 1983;113:173-215. http:// dx.doi.org/10.1016/0165-1161(83)90010-9

28. Buschini A, Pinelli S, Pellacani C, Giordani F, Ferrari MB, Bisceglie F, et al. Synthesis, characterization and deepening in the comprehension of the biological action mechanisms of a new nickel complex with antiproliferative activity. J Inorg Biochem. 2009;103:666-77. http://dx.doi. org/10.1016/j.jinorgbio.2008.12.016

29. Kanupriya, Prasad D, Sai Ram M, Kumar R, Sawhney RC, Sharma SK, et al. Cytoprotective and antioxidant activity of Rhodiola imbricata against tert-butyl hydroperoxide induced oxidative injury in U-937 human macrophages. Mol Cell Biochem. 2005;275:1-6. http://dx.doi.org/10.1007/s11010005-7637-1

30. Lacoste S, Castonguay A, Drouin R. Formamidopyrimidine adducts are detected using the comet assay in human cells treated with reactive metabolites of 4-(methylnitrosamino)1-(3-pyridyl)-1-butanone (NNK). Mutat Res. 2006;600:13849. http://dx.doi.org/10.1016/j.mrfmmm.2006.04.005

31. Hartmann A, Agurell E, Beevers C, Brendler-Schwaab S, Burlinson B, Clay P, et al. Recommendations for conducting the in vivo alkaline comet assay. 4th International Comet Assay Workshop. Mutagenesis. 2003;18:45-51. http:// dx.doi.org/10.1093/mutage/18.1.45

32. Hartmann A, Schumacher M, Plappert-Helbig U, Lowe P, Suter W, Mueller L. Use of the alkaline in vivo comet assay for mechanistic genotoxicity investigations. Mutagenesis. 2004;19:51-9. http://dx.doi.org/10.1093/mutage/geg038

33. Tice R, Vásquez M. Protocol for the application of the $\mathrm{pH}>13$ alkaline single cell gel (SCG) assay to the detection of DNA damage in mammalian cells-1999. Fecha de consulta: 5 de noviembre de 2011. Disponible en: http://cometassay.com/ Tice\%20and\%20Vasques.pdf.

34. Tice RR, Agurell E, Anderson D, Burlinson B, Hartmann A, Kobayashi H, et al. Single cell gel/comet assay: Guidelines for in vitro and in vivo genetic toxicology testing. Environ Mol Mutagen. 2000;35:206-21. http://dx.doi.org/10.1002/ (SICI)1098-2280(2000)35:3<206::AID-EM8>3.0.CO;2-J

35. Maistro EL, Carvalho JC, Mantovani MS. Evaluation of the genotoxic potential of the Casearia sylvestris extract on HTC and V79 cells by the comet assay. Toxicol In Vitro. 2004;18:337-42. http://dx.doi.org/10.1016/j.tiv.2003.10.002

36. Palus J, Dziubaltowska E, Rydzynski K. DNA damage detected by the comet assay in the white blood cells of workers in a wooden furniture plant. Mutat Res. 1999;444:6174. http://dx.doi.org/10.1016/S1383-5718(99)00089-3

37. Wang H, Ma C, Ma L, Du Z, Ye H, Li G, et al. Secondary metabolic profiling and artemisinin biosynthesis of two genotypes of Artemisia annua. Planta Med. 2009;75:162533. http://dx.doi.org/10.1055/s-0029-1185814

38. Wink M. Evolution of secondary metabolites from an ecological and molecular phylogenetic perspective. Phytochemistry. 2003;64:3-19. http://dx.doi.org/10.1016/ S0031-9422(03)00300-5

39. Friedman M, Lee KR, Kim HJ, Lee IS, Kozukue N. Anticarcinogenic effects of glycoalkaloids from potatoes against human cervical, liver, lymphoma, and stomach cancer cells. J Agric Food Chem. 2005;53:6162-9. http:// dx.doi.org/10.1021/jf050620p

40. Nagai F, Hiyoshi Y, Sugimachi K, Tamura HO. Cytochrome P450 (CYP) expression in human myeloblastic and lymphoid cell lines. Biol Pharm Bull. 2002;25:383-5. http://dx.doi. org/10.1248/bpb.25.383 